\title{
Ética del cuidado de sí y desarrollo humano: un reto para la Educación Superior
}

\author{
Gladis del Socorro García Restrepo*
}

\begin{abstract}
Resumen
Los cursos universitarios de desarrollo humano son vistos por algunos actores del sistema educativo como apéndices sin sentido en las mallas curriculares. Lo que pretende mostrar este escrito es que son altamente relevantes al momento de pensar una sociedad más justa y humana, y que en el propósito de desentrañar su sentido el pensamiento de Foucault podría servir de acicate, en tanto sus reflexiones sobre el cuidado de sí entendido por él como un asunto ético, dado que conlleva relaciones con los otros, coincide con algunos objetivos trazados en dichos cursos; por tanto, se mostrará que la dualidad desarrollo humano y cuidado de sí se complementan.
\end{abstract}

Palabras clave: desarrollo humano, cuidado de sí, ética, currículo.

\section{Self-care ethics and human development: a challenge for Higher Education}

\begin{abstract}
The university courses about human development are regarded, by some of the actors involved in the education system, as insignificant appendixes in the curricula. This paper aims to demonstrate their relevance at the moment of thinking about a more just and human society and, in the purpose to unveil its significance, Foucault's thinking could be an incentive given his reflections about self-care, as he understands it as an ethical issue because it includes
\end{abstract}

the relationships with others. This matches some of the objectives those courses have and, therefore, the complementary relationship between human development and self-care will be demonstrated.

Key words: human development, self-care, ethics, curriculum.

\section{Ética do cuidado de si e desenvolvimento humano: um desafio para a educação superior}

\section{Resumo}

Os cursos universitários de desenvolvimento humano são vistos por alguns atores do sistema educativo como apêndices sem sentido nas grades curriculares. O que pretende mostrar este escrito é que são altamente relevantes ao momento de pensar uma sociedade mais justa e humana, e que no propósito de desentranhar seu sentido o pensamento de Foucault poderia servir de acicate, em tanto suas reflexões sobre o cuidado de si entendido por ele como um assunto ético, dado que implica relações com os outros, coincide com alguns objetivos traçados em ditos cursos; por tanto, se mostrará que a dualidade desenvolvo humano e cuidado de si se complementam.

Palavras importantes: desenvolvimento humano, cuidado de si, ética, currículo.

\footnotetext{
* Bacterióloga y laboratorista clínica. Especialista en Gerencia de Servicios de Laboratorio Clínico de la Universidad de Antioquia. Magíster en Educación y Desarrollo Humano de la Universidad de Manizales. Doctorando en Filosofía Universidad Pontificia Bolivariana. Docente de la Escuela de Microbiología - Universidad de Antioquia.
} 


\section{Introducción}

El caótico panorama al cual asiste el hombre contemporáneo reclama de las instituciones de Educación Superior una permanente reflexión en torno a su responsabilidad social en términos del desarrollo humano de sus egresados. De ahí que sea recurrente observar en las declaraciones misionales de las universidades el compromiso con el desarrollo humano o humanístico de estudiantes, egresados y demás estamentos, intención que, además de comprensible, es necesaria. Ortega y Gasset, en Misión de la Universidad, llamaba la atención sobre la necesidad de humanizar particularmente a los universitarios, quienes una vez graduados entrarían a aportar desde su saber a la sociedad; al respecto señalaba: "Hay que humanizar al científico" (1968, p. 67). En tal sentido la universidad, dada su razón de ser soportada en su historicidad, no puede desarrollarse desvinculada de las necesidades fundamentales de la sociedad y una de esas necesidades está relacionada con el precario sentido de humanidad que exhiben algunas de sus instituciones, situación que se ve reflejada en el caos social, económico y ambiental, visibilizado en los diferentes ámbitos del accionar humano.

Los desarrollos disciplinares de cada programa académico no deberían alejarse del sentido de humanidad que generalmente los sustenta; por el contrario, deberían fortalecerse mutuamente, dado que al no interiorizarse la dualidad humanismo-ciencia, se estaría propiciando desde el aula lo que también advertía Zambrano: "El pensamiento científico, descualificador, desubjetivador anula la heterogeneidad del ser, es decir, la realidad inmediata, sensible" (1989, p. 68). Es en este contexto donde se encuentra pertinente el ejercicio de la ética del cuidado de sí, pensada por Foucault tomando como referente la cultura greco-romana, dado que su conexión con las cátedras enfocadas al desarrollo humano podría contribuir a que los estudiantes las hallaran, además de pertinentes, dotadas de sentido para su ejercicio pro- fesional y su dinámica vital expresada en las distintas dimensiones de su ser.

Para el desarrollo de la presente reflexión se plantearán tres subtemas: en el primero se harán algunos planteamientos respecto a la subvaloración de las cátedras de desarrollo humano por parte de ciertos sectores sociales; en segundo lugar se abordarán determinados elementos clave respecto a la ética del cuidado de sí y el cuidado de los otros desde la perspectiva foucoultiana, y por último, se mostrará cómo estos elementos podrían complementar y re-significar las intencionalidades curriculares referidas al desarrollo humano.

\section{Currículo y desarrollo humano: algunos planteamientos}

Reflexionar acerca de los cursos de desarrollo humano en el plano universitario es un asunto que de inmediato remite al terreno del sujeto en tanto el desarrollo de su ser; a su vez, es la oportunidad para pensar el humanismo desde la praxis y la teoría, en razón a que el propósito tácito o expreso de las comunidades académicas que diseñan los currículos es dar cumplimiento a las directrices misionales propias de algunas instituciones educativas, en las que se proclama su compromiso con tal categoría, pero ¿qué se entiende por humanismo? La respuesta a este interrogante es compleja y extensiva, dado que puede darse desde diferentes perspectivas políticas y de pensamiento, y ubicarse en determinados momentos históricos; en tal sentido, es comprensible por qué muchas de las concepciones sobre este tema sigan siendo susceptibles de fuertes críticas; de ahí que el mismo Foucault expresara cierto sigilo al pronunciarse al respecto, tal como pudo observarse en una entrevista concedida el 25 de octubre de 1982 -a raíz del revuelo que causó en la comunidad académica* la publicación de Las palabras y las cosas- en la que advertía:

\footnotetext{
* $\quad$ En la introducción a Las palabras y las cosas del año 1985 aparece el siguiente comentario: "En 1966, la publicación de Las palabras y las cosas dio lugar, en los medios intelectuales franceses de la época, a un polémica de considerables proporciones (...) El tono de Foucault era, desde luego, provocador. Enfrentándose a la tradición del humanismo, en la forma como se ha articulado en la cultura europea desde el Renacimiento hasta nuestro días" (Foucault, 1985).
} 
Lo que me asusta del humanismo es que presenta cierta forma de nuestra ética como modelo universal para cualquier tipo de libertad. Me parece que hay más secretos, más libertades posibles y más invenciones en nuestro futuro de lo que podemos imaginar en el humanismo (Foucault, 1991, p. 150).

Según esto el humanismo, para serlo, no puede acomodarse a determinada ideología o servir a ciertos propósitos políticos; su teorización y su práctica tampoco son estáticas porque la historia del hombre y de la sociedad tampoco lo es.

En este mismo sentido expresaba Hoyos: "En su Carta sobre el humanismo Martín Heidegger en 1946, inmediatamente después de la guerra, critica toda forma de humanismo, en especial el cristiano, el marxista y el existencialista, por pretender dar razón de la existencia humana, sin tener en cuenta el sentido mismo del existir (Dasein)" (2009, p. 429) y más adelante agrega: "Se hace pues necesario rescatar un paradigma humanista para la educación contemporánea, pero tomando el humanismo en su forma más amplia y compleja como propósito y como programa" (p. 430).

Estos y muchos otros pronunciamientos respecto al humanismo conducen a reflexionar sobre lo que pensadores como Derrida* han llamado nuevo humanismo a la hora de pensar la universidad: "El nuevo humanismo que estamos buscando es el de Universidad sin condición (gracias a las "humanidades"), en el sentido propuesto por Jacques Derrida en Stanford en abril de 1998 y de nuevo en FranKfurt en 2000 y en Murcia un año después" (Hoyos, 2009, p. 430).

¿Por qué traer a la reflexión los anteriores planteamientos? Porque los sistemas educativos, por lo menos el colombiano, hacen parte de la estructura del Estado; esto explica en buena medida por qué las reformas educativas generalmente quedan insertas en la filosofía que subyace a las políticas y tendencias del Gobierno correspondiente a determinado momen- to histórico; de ahí que sea menester retornar a la invitación hecha por Derrida. No obstante, esta invitación cada día está más lejana de la realidad, en razón a que se observa de manera dramática cómo van desapareciendo de las mallas curriculares los cursos que intentan darle vía a este propósito, al respecto Nussbaum (2012) manifiesta:

En casi todas las naciones del mundo se
están erradicando las materias y las ca-
rreras relacionadas con las artes y las hu-
manidades, tanto a nivel primario y secun-
dario como a nivel terciario y universitario.
Concebidas como ornamentos inútiles por
quienes definen las políticas estatales en
un momento en que las naciones deben
eliminar todo lo que no tenga ninguna uti-
lidad para ser competitiva en el mercado
global, estas carreras y materias pierden
terreno a gran velocidad, tanto en los pro-
gramas curriculares como en la mente y el
corazón de padres e hijos (p. 20).

La idea de "ornamentos inútiles" ha ido calando de manera paulatina en los diferentes estamentos de la sociedad y más específicamente en el sector educativo, al punto que cuando se entra en esas inexplicables transacciones de créditos a fin de que los planes de estudio se ciñan a la ley del momento o por cualquier otra circunstancia, con recurrencia se trasladan créditos de las áreas humanísticas a las disciplinares, sin ninguna o poca reflexión al respecto $y$, lo que es peor, en nombre de las reformas curriculares se exhiben argumentos para transformar los currículos pero esa transformación a lo que apunta, en muchos casos, es a hacer desaparecer las cátedras de orden humanístico. Situaciones como esta deberían de alertar a los actores del sistema educativo, y remitirlos a una profunda reflexión, en tanto negociar las cátedras humanísticas de un currículo es negociar, en cierta medida, el sentido de humanidad de la universidad y, por tanto, de la sociedad a la que se debe.

Por lo anterior, es menester aprovechar el espacio universitario para debatir asuntos como el relacionado con el papel de las humanida-

\footnotetext{
"Allí dijo que la Universidad del futuro debería ser totalmente libre: en ella no debería obstaculizarse de ninguna forma la investigación (...) Las preguntas orientadoras que habría que considerar en esta universidad, deberían ser por ejemplo, las preguntas por los derechos humanos, la diferencia de género, el racismo (...) En esta universidad hay que trabajar filosóficamente. Se desean análisis de conceptos, pero también resistencia" (Hoyos, 2009, p. 430).
} 
des en la formación de los nuevos profesionales y en la dinámica social, y volver una y otra vez a lo proclamado en las misiones institucionales respecto al humanismo, a fin de que no sea letra muerta y reflexionar, de paso, en lo que Ortega y Gasset (1964) advertía: "Es preciso que el hombre de ciencia deje de ser lo que hoy es con deplorable frecuencia: un bárbaro que sabe mucho de una cosa" (p. 68). Avanzando en esta reflexión es oportuno traer nuevamente a Nussbaum (2005) cuando en su libro El cultivo de la humanidad propone en esta misma línea, tres habilidades para cultivar la humanidad en el mundo actual: la primera es la referente a la "(...) habilidad para un examen crítico de uno mismo y de las propias tradiciones, que nos permita experimentar lo que, siguiendo a Sócrates, podríamos llamar 'vida examinada"' (p. 28); en la segunda llama la atención sobre la "(...) capacidad de verse a sí mismo no solo como ciudadanos pertenecientes a alguna región o grupo, sino también, y sobre todo, como seres humanos vinculados a los demás seres humanos por lazos de reconocimiento y mutua preocupación" (p. 29), y la tercera "(...) puede llamarse imaginación narrativa. Esto significa la capacidad de pensar cómo sería estar en el lugar de otra persona, ser un lector inteligente de la historia de esa persona, y comprender las emociones, deseos y anhelos que alguien así pudiera experimentar" (p. 30).

En la adquisición de las habilidades señaladas por Nussbaum entran en juego diversas variables y escenarios, pero sin duda la educación, como vía para que el ser humano se reconozca como proyecto en vía de llegar a ser, se entienda como un ser en relación y desarrolle en toda su significación el sentido de solidaridad, juega un papel relevante en todos y cada uno de sus niveles y contextos.

\section{La ética del cuidado de sí y de los otros}

Las reflexiones que hace Foucault desde su perspectiva genealógica de epiméleia heautou, que significa el cuidado de uno mismo (1994, p. 34 ), tienen como referente la cultura griega*, e implican una serie de actividades que el sujeto emprende en el marco de su reflexividad con el propósito de volver su mirada sobre sí mismo, sus pensamientos, sus actuaciones y su relación con los otros y que el autor ha denominado Tecnologías del yo, las cuales le posibilitan

(...) efectuar, por cuenta propia o con la ayuda de otros, cierto número de operaciones sobre su cuerpo y su alma, pensamientos, conducta, o cualquier forma de ser, obteniendo así una transformación de sí mismos, con el fin de alcanzar cierto estado de felicidad, pureza, sabiduría e inmortalidad (1991, p. 48).

Se resalta en el anterior planteamiento el propósito de la transformación personal como también lo relevante de la presencia del otro en el logro de tal objetivo; de ahí que para Foucault el cuidado de sí sea un asunto ético, respecto al cual plantea: "El cuidado de sí es ético en sí mismo: pero implica relaciones complejas con los otros, en la medida en que este ethos ${ }^{* *}$ de la libertad es también una manera de ocuparse de los otros" (1994, p. 116). En esta línea es preciso recordar que para la cultura grecoromana la idea de libertad estaba directamente relacionada con "la no-esclavitud -lo que constituye sin duda una definición de la libertad bastante alejada de la nuestra-, el problema es un problema totalmente político" (p. 115). Según el pensamiento foucaultiano el asunto es de orden político dado que la "condición" de ser esclavo impediría de suyo el ejercicio de la libertad y consecuentemente el ejercicio de la ética, lo que en palabras del mismo autor sería: "(...) un esclavo no tiene ética" (p. 116).

De acuerdo con Foucault (1994) la libertad es un asunto político, pero él va más allá en su reflexión y señala: "(...) ser libre significa no ser esclavo de sí mismo ni de los propios apetitos, lo que implica que uno establece en relación consigo mismo una cierta relación de

\footnotetext{
* El precepto 'ocuparse de uno mismo' era, para los griegos, uno de los principales principios de las ciudades, una de las reglas más importantes para la conducta social y personal y para el arte de la vida" (Foucault, 1991, p. 50).

** "El ethos de alguien se expresaba a través de su forma de vestir, de su aspecto, de su forma de andar, a través de la calma con la que se enfrentaba a cualquier suceso (...) implica también una relación para con los otros, en la medida en que el cuidado de sí convierte a quien lo posee en alguien capaz de ocupar en la ciudad, en comunidad, o en las relaciones interindividuales, el lugar que conviene" (Foucault, 1994, p. 115-116).
} 
dominio, de señorío, que se llamaba arché, poder mando" (p. 116). En tal sentido el hecho de ser libre implica, entre otras cosas, ejercer un cierto dominio sobre sí mismo, intención en la que tiene un significativo valor la mirada crítica con la que se aborde no solo la propia realidad sino también la circundante; realidad en la que pulula un gran número de situaciones en las que es fácil que el individuo quede atrapado, ya que ejercen sobre él otro tipo de esclavitud, que si bien en algunos aspectos es lejana a la padecida en la cultura greco-romana, en otros sigue siendo similar, en razón a que aparecen otros amos tales como el consumismo, que dictará qué comprar, qué cuidar, qué no cuidar, cómo comportarse, qué pensar, qué estudiar, en qué trabajar y hasta cómo relacionarse con los otros; contexto en el que nuevamente podría pensarse que el hecho de no ser ni sentirse libre conllevaría el no ser ético. En este contexto y refiriéndose al sentido de la libertad Foucault señala “(...) para que esta práctica de la libertad adopte la forma de un ethos que sea bueno, bello, honorable, estimable, memorable, y que pueda servir de ejemplo, es necesario todo un trabajo de uno sobre sí mismo" (1994, p. 115).

Se intuye a partir de la anterior reflexión que hacer una apuesta por el ejercicio de una ética del cuidado de sí es, entre otras cosas, una estrategia en virtud de la cual es posible que el hombre se proponga anular desde la manera como vive, los diversos flagelos que lo afectan $y$, a su vez, perturban la sociedad, los cuales emergen y dimensionan debido a su empobrecido sentido de humanidad, situación a la que lamentablemente no escapa el sistema educativo; por el contrario, en ocasiones es quizá el instrumento más eficiente a la hora de perpetuar en las sociedades ciertos modos de abordar la realidad y conservar determinados arquetipos de comportamiento, acciones que de suyo facilitan a ciertos sectores de la sociedad dirigir el modo de vivir de una colectividad, que cada vez es más global que local. No obstante, la educación también podría ser vista como una vía a través de la cual se motive al estudiante para que le dé un giro a su mirada sobre el mundo y sobre sí mismo, claro está en un ambiente de profundo respeto por sus principios y convicciones, pues de acuerdo con Cubides (2006) "La educación consiste en contribuir positivamente para que, respetando la diferencia personal del educando, éste vaya siendo artífice de su propia formación y se convierta en un interlocutor válido" (p. 117).

\section{La ética del cuidado de sí y los cursos enfocados al desarrollo humano}

Para dar inicio a este aparte es pertinente preguntarse ¿por qué los cursos orientados al desarrollo humano son vistos por algunos actores del sistema educativo como "ornamentos inútiles"? Al tratar de dar respuesta a este interrogante se encuentran algunos planteamientos como el de Orozco quien al referirse a las limitaciones concernientes a los conocimientos humanísticos manifiesta: "(...) una vez hechos los cursos por parte del estudiante, el fin se considere alcanzado; olvidar que por naturaleza este tipo de formación es más el logro de un enfoque que afecta todos los currículos y que cualifica las tareas sustantivas de la institución" (2002, p. 35). Según este autor, el hecho de que el estudiante apruebe estos cursos no lo hace automáticamente ni más humano ni más ético; no obstante, lo que se esperaría es que lo vivido en el aula le otorgue pistas para aproximarse al mundo de tal modo que vaya marcando en él una tendencia, ojalá ética y humana, que permee su vida en todas sus esferas y lo proyecte al futuro como un profesional idóneo. En esta intencionalidad no pueden desfallecer las instituciones educativas; por el contrario, sus actores deben acudir a las estrategias que les sean posibles, para permear de esta filosofía los distintos ámbitos de la vida universitaria.

De otro lado, Nussbaum (2005) señala: “(...) la educación para el crecimiento económico se opondrá a la presencia de las artes y las humanidades como ingrediente de la formación elemental mediante un ataque que, hoy en día, se puede observar en todo el planeta" (pp. 4647). Esta advertencia es clara, máxime si se considera que el ambiente neoliberal en el que se desarrolla la sociedad actual lo que intenta, entre otras cosas, es abolir el sentido crítico con que el hombre, en este caso concreto el estudiante universitario, aborda la realidad, a fin de hacer de él y su profesión un objeto más 
en el caótico panorama mercantil y consumista en el que se encuentra inmerso y en el que priman, por encima de todo, los indicadores económicos que pueda alcanzar cada nación, ámbito en el que es factible la anulación de todo esbozo de humanismo y eticidad.

De otro lado es oportuno tener en cuenta que "Si se forman docentes, verdaderos intelectuales, creativos, participativos, etc., el efecto en el sistema educativo se notará para su beneficio; pero también se notará en el caso contrario" (Vargas, 1993, p. 29). De acuerdo con esta consideración si el docente está mejor preparado y canaliza sus esfuerzos para ir construyéndose como un intelectual ${ }^{*}$, podrá abordar los temas de contenido humanístico o los que le correspondan, desde un ángulo de mayor pertinencia, objetividad y convicción; igualmente si exhibe en su práctica docente un buen dominio de las diversas estrategias para comunicar tal saber, sin duda tendrá más posibilidades de alcanzar los objetivos académicos propuestos, haciendo que tales cátedras vayan perdiendo el estigma de "ornamentos inútiles".

El objeto de estudio en torno al cual se construye un programa académico, también es relevante a la hora de que estudiantes y profesores vayan en busca del sentido de las cátedras de contenido humanístico, pues las discusiones que se propongan en torno a este servirán no solo para aproximarse aún más a su compresión sino también a su proyección en términos de lo humano y lo humanizante; en tal sentido argumenta Vargas (1993): "Enseñar un saber, mostrar su objeto de estudio y el respectivo régimen de procedimiento con respecto al mismo en su historicidad requiere un contexto enmarcante y, hasta cierto punto, esencial: el proyecto de ser humano, de sociedad y de cultura en pro del cual se ordena" (p. 30).

En el marco de las anteriores consideraciones y en el intento por re-significar las cátedras enfocadas al desarrollo humano desde la ética del cuidado de sí, es preciso reconocer lo que advierte Cubides: "Si bien Foucault reconoce la imposibilidad de hallar la solución de un problema como lo hubiera resuelto gente de otra época, no obstante cree que la noción de cuidado de sí puede adoptar un sentido actual" (2006, p. 121). Es claro que no es suficiente con mirar hacia el pasado para encontrar soluciones en el presente, pero también es cierto que tal ejercicio permite mostrar que el cuidado de sí “(...) es un principio o noción creada en la Antigüedad para denotar múltiples sentidos, entre ellos la idea de un trabajo que implica atención, conocimientos y técnicas, de una actividad dirigida a la conversión de uno mismo por sí mismo, a la elaboración de un arte de sí y de un arte de vivir" (Cubides, 2006, p. 121); de otro lado, las cátedras de desarrollo humano lo que intentan, entre otras cosas, es que el futuro profesional se visualice y se prepare para desempeñarse en escenarios laborales, en los que tendrán tanto valor sus saberes disciplinares como su accionar ético y humano: se intuye entonces que la praxis de lo que Foucault nombró "tecnologías del yo" podrían potenciar y por tanto re-significar las cátedras de desarrollo humano, dado que si el estudiante logra o procura hacer de su vida una obra de arte en tanto el cuidado que se prodiga a sí mismo, a fin de estar atento a su modo de ser y actuar en el mundo, gracias a la reflexión permanente que tiene sobre sus actos, con certeza le quedará más fácil comprender que debe proyectarse hacia los otros y lo otro, prodigándoles un cuidado similar, en virtud de que también son su responsabilidad. Comprenderá, además, que la utopía de transformar otros escenarios es posible, gracias a la posibilidad de transformar su propio universo, acciones que de suyo conllevan la construcción de un alto sentido de humanidad en lo personal y lo colectivo; de ahí que del cuidado de sí se desprenda un carácter eminentemente ético en razón al sentido proyectivo que lo sustenta.

Son muchas las estrategias que se pueden desplegar en el aula para conectar el cuidado de sí con las cátedras de desarrollo humano;

"El intelectual podría pensarse como el hombre formado, el hombre ético, el hombre educado en este preciso sentido del ideal de la Bildung; no educado en el sentido que conduzca a otro, o que produzca un saber, en el sentido de hacer producir un aprender, una capacidad o competencias. El intelectual no establece relaciones como la del profesor y el alumno o padre e hijo o adulto-niño. El intelectual es un estado, una experiencia, un estilo de vida que se preocupa de producir libertad, de luchar por la libertad, de crear libertades no para otros, sino para sí mismo, para su propio ser como sujeto" (Quiceno, 2003, p.215). 
una de ellas es el ejercicio de la lectura*, dado el significativo valor que tiene en los procesos de enseñanza-aprendizaje y, por supuesto, en el contexto investigativo, pues la lectura como estrategia no solo se refiere a la lectura de textos escritos, sino que es un ejercicio que también conecta con la lectura de la realidad como texto posible, en la que es factible leerse y leer el accionar humano desde diferentes ángulos y perspectivas; por tanto, es de esperarse que la lectura de textos académicos, sumada a la lectura de la realidad dinamicen el desarrollo humano y, a su vez, la práctica científica, pues a partir de este ejercicio es altamente probable que surjan preguntas dirigidas no solo hacia sí mismo sino también hacia el entorno, actitud que con certeza alimentará las inquietudes en el campo investigativo.

En esta intención de leer, leerse y leer la realidad, cobra significativa importancia la participación de la comunidad académica en las actividades que se derivan de los diferentes planes de cultura o de bienestar universitario que tienen algunas universidades, dado que a través de estas es posible el acercamiento al arte en sus diferentes manifestaciones. La vía del arte como texto es importante, entre otras cosas, porque es la posibilidad para que el estudiante a través de sí mismo, vuelva reflexivos verbos como narrar, describir, mirar, proponer, criticar, observar, escuchar, entre otros, de tal forma que le sea posible narrarse (yo me narro), describirse (yo me describo), mirarse (yo me miro), criticarse (yo me critico), observarse (yo me observo), escucharse (yo me escucho), actividad que lo estimulará en su intento por perfilarse como sujeto que se construye críticamente, que se proyecta como un ser humano que comprende que cualquier intento de transformación de la realidad deberá iniciar por su propia transformación y que la humanización, en tanto actitud ética en el obrar, dejará de ser una utopía cuando tal ideal opere en su propio mundo. En síntesis, la conjugación armónica de la ética del cuidado de sí con las temáticas desarrolladas en las cátedras de de- sarrollo humano, dirigidas a permear el resto del currículo, permitirá proyectar una formación con tendencia a la integralidad y, por tanto, al humanismo y a la ética como modo de ser en la existencia.

\section{Conclusiones}

Las cátedras universitarias enfocadas al desarrollo humano $y$, por supuesto, las artes y las ciencias sociales y humanas, no son "ornamentos inútiles"; por el contrario, son de alta relevancia a la hora de proyectar una sociedad más justa y humana; por lo tanto, es menester que el sector educativo explore otros caminos con la intención de hallar nuevas claves que les permitan a los estudiantes y a la comunidad académica, encontrarle sentido a aquel saber que ha sido encasillado en las denominadas humanidades, en las que aparecen, entre otras, cátedras como la ética y la bioética, cuyo propósito es procurar un mejor estar con los otros, lo otro y consigo mismo, y es ahí precisamente donde lo planteado por Foucault en términos de "la ética del cuidado de sí" puede de modo sustantivo potenciar y dimensionar dichas cátedras, dado que lo que se esperaría es que el accionar ético y humano de los egresados universitarios sea producto del permanente discernimiento sobre sí mismos y sobre la realidad que los circunda, a fin de que en su proyección a la sociedad intenten permanentemente "cultivar la humanidad".

\section{Referencias bibliográficas}

- Cubides, H. (2006). Foucault y el sujeto político: ética del cuidado de sí. Bogotá: Siglo del Hombre Editores.

- $\quad$ Foucault, M. (1985). Las palabras y las cosas. Barcelona: Siglo XXI Editores, S. A.

- Foucault, M. (1991). Tecnologías del yo y otros textos afines. Barcelona: Paidos Ibérica, S.A.

- Foucault, M. (1994). Hermenéutica del sujeto. Madrid: Ediciones Endymion.

“(...) leer y enseñar a leer son prácticas esencialmente implicadas en eso que llamamos educación. No sólo porque gran parte de las actividades educativas se dan en la lectura, a través de la lectura, sino también porque enseñar a leer es uno de los objetivos principales de toda educación. Las prácticas educativas modernas son prácticas letradas y son también prácticas a través de las cuales los individuos son iniciados en la cultura letrada. Pero el lenguaje es más, y otra cosa, que un vehículo de la educación. Y enseñar a leer es más, y otra cosa, que alfabetizar" (Larrosa, 2008, p. 291). 
- Hoyos, G. (2008). Filosofía de la educación. En: Larrosa. Leer (y enseñar a leer), entre las lenguas. Veinte fragmentos (y muchas preguntas) sobre lenguaje y pluralidad. Madrid: Editorial Trotta. pp. 277-292.

- Hoyos, G. (2009). Educación para un nuevo humanismo. Magis, 1(2), 425-433.

- Nussbaum, M. (2005). El cultivo de la humanidad. Barcelona: Edicones Paidós Ibérica, S. A.

- Nussbaum, M. (2012). Sin fines de lucro. Buenos Aires: Katz Editores.
- Orozco, L. (2002). La formación integral como base para definir estrategias de un pensamiento lúcido y pertinente. Debates (32), 34-43.

- Ortega y Gasset, J. (1968). Misión de la Universidad y otros ensayos afines. Madrid: Revista de Occidente.

- Quiceno, H. (2003). Michael Foucault, ¿pedagogo? Educación y Pedagogía, 15(37), 201-216.

- Vargas, G. (1993). Formación y subjetividad. Epistemología, lenguaje y pedagogía. Educación y Pedagogía, 4 (98), 17-37.

- Zambrano, M. (1989). Senderos. Barcelona: Editorial Anthropos. 